\title{
Molecular Docking of Isorhamnetin as CYP1A1 Inhibitor in Skin Photoaging
}

\author{
1 \\ Izzatul Lailiyah, Aulanni'am*, Sasangka Prasetyawa \\ Department of Chemistry, Faculty of Science, Brawijaya University \\ Faculty of Veterinary Medicine, Brawijaya University \\ *Correspondent author: aulani@ub.ac.id
}

\begin{abstract}
Skin aging is the aging process of skin tissue due to elastin and collagen breakdown. Collagen and elastin are protein of connective tissue in skin dermis that serves to regenerate the skin for firmness and flexibility maintained. Sunlight is composed by three main parts according to their wavelengths, namely UVA, UVB, and UVC where the intensity of UVB sunlight is most active. UVB generates the production superoxide anion $\left(\mathrm{O}^{2-}\right)$ which is main free radical in the skin surface. It attacked the cell membrane and subsequently form a new ROS and decrease antioxidants enzymatic excesively. Excessive ROS production resulting overexpression of AP-1 which activated Matrix metalloproteinases lead to collagen breakdown and photoaging. This paper discusses the affinity and interaction of isorhamnetin to CYP1A1 causing inactivation of AP-1 using molecular docking program. CYP1A1 is protein member of cytochrome P450 superfamily of enzymes encoded by the CYP1A1 gene. Cytochrome P450 used as oxidazing catalist in metabolic pathways steroids, fatty acids, xenobiotics, including drugs, toxins and carcinogens. This study consist of two stages: (1) isorhamnetin-CYP1A1 docking and $\alpha$-naphthoflavone-CYP1A1 redocking (2) $\Delta G$ scores, inhibitory constant, and bonding interaction between ligan-reseptor analysis. Gibbs energy and inhibitory $(\Delta G)$ constant (Ki) showed stability interaction between isorhamnetin and CYP1A1. Based on the $\Delta G$ score isorhamnetin has higher potential as CYP1A1 inhibitor than-naphthoflavonea. Isorhamnetin-

CYP1A1 get $-10,3 \mathrm{kcal} / \mathrm{mol}$ and $11,34 \mu \mathrm{M}$ and-naphthoflavone $\alpha$ get $-9,1 \mathrm{kcal} / \mathrm{mol}$ and $228,4 \mu \mathrm{M}$. Isorhamnetin-CYP1A1 binded by $4 \pi$ bondingand2hydrogen bonding. The result presented the potential of isorhamnetin to decreased AP-1 expressioan through CYP1A1 inhibition.
\end{abstract}

Keyword: photoaging, AP-1, isorhamnetin, CYP1A1, molecular docking

\section{INTRODUCTION}

Skin aging (skin aging) is the aging process of skin tissue due to decreased levels of collagen and elastin in skin'scells. Collagen and elastin are the protein of connective tissue in skin dermis that serves to regenerate skin so that the firmness and flexibility is maintained 4 Aging (aging) can occur on the skin, caused by internal factors or genetic (chronological aging) and external factors, namely ultraviolet rays (photoaging) ${ }^{2}$. UVB triggers the production of superoxide anion $\left(\mathrm{O}^{2-}\right)$ primarily through activation of NADPH oxidase and mitochondrial respiratory chain reaction which is then converted into ${ }^{1} \mathrm{O}_{2}$. Singlet oxygen which is the main ROS in the skin surface attacked the cell membrane and forms a new ROS subsequently. The higher the accumulation of ROS will affected the processes of skin damage particularly collagen. UVB activated TGF- $\beta$ receptor directly and lowering Retinoic Acid (RA) which is an inhibitor of AP-1 pathway. It will activated NFkB and AP-1 and increases levels of ROS. Accumulations of ROS also increase the number of proinflammatory cytokines and growth factors. This helps activated AP-1 pathways that function in the regulation of MMPs and procollagen. AP-1 is a transcription factor that plays a role in activating matrix metalloproteinases whereas responsible for the degradation of collagen. In the end, the accumulation of excess ROS causing reduced production of collagen and 
collagen break down that lead to photoaging. UV is also able to directly reduce the amount of TGF- $\beta$ and regulate NF-kB pathway that will let go of proinflammatory cytokines ${ }^{3}$.

Cytochrome P450 is an enzyme composed of heme and cofactors, so it is also called hemeprotein, lies in the mitochondrial membrane or in endoplasmic reticulum. Cytochrome P450 (CYP) in almost all tissues and play an important role in the synthesis of hormones, cholesterol synthesis, and vitamin D metabolism. In addition, CYP also contribute to the metabolism of toxins, drugs, and other endogenous metabolic products. Cytochrome P50 is one of the ROS producers where found in the endoplasmic reticulum. This process began with the transfer of NADP oxidase to CYP via CYP reductase which results in xenobiotic hydroxylation. Cytochrome P450 is divided into several families depends on the gene that code for CYP. The release of electrons during this transfer process causes free radicals 1 .

\section{METHODS}

Molecular docking of CYP1A1 and isorhamnetin using some molecular programs that was DS. Visualisixer 2016, PyMOL, PyRX, docking server and stitch.embl.de to showed AP1 and CYP1A1 corellation. Collected CYP1A1 encoded from uniprot.org and modeling using swissmodel.expasy.org to get the CYP1A1 3D structure as receptor protein. Collected isorhamnetin 3D structure from pubchem.ncbi.nlm as ligand and was doing docking using PyMOL. Visualization of docking result such as $\Delta \mathrm{G}$ scores, inhibitory constant, and bonding interaction between ligan-reseptor were used by DS. Visualisixer 2016.

\section{RESULT}

Isorhamnetin is methylated metabolite of quercetin with in vitro antioxidant activity. Isorhamnetin acts as ligan in these studies to inhibit CYP1A1 activity. Ligand-receptor conformation that chosen is the lowest energy conformation. $\Delta \mathrm{G}$ scores is the energy released during the process of ligand-receptor binding. Ligand-receptor interaction

tends to be at its lowest energy condition because it because the molecule state be stable so the smaller the $\Delta \mathrm{G}$ scores the stable the structure formed. In addition, the data obtained inhibition constants $(\mathrm{Ki})$ which the ligand can be bound stronger when Ki gets smaller. Based on the analysis of the $\Delta \mathrm{G}$ scores results showed isorhamnetin-CYP1A1 is- $10.3 \mathrm{kcal} / \mathrm{mol}$, smaller than-naphthoflavone $\alpha$-CYP1A1 amounted $-7.8 \mathrm{kcal} / \mathrm{mol}$-naphthoflavone. $\alpha$ is a drug ligand

which binding with CYP1A1 (no.PDB: 4i8v). Isorhamnetin-CYP1A1 Ki value is $11.34 \mu \mathrm{M}$ smaller-naphthoflavonethan-CYP1A1 $\alpha$ amounted $228.4 \mu \mathrm{M}$. 


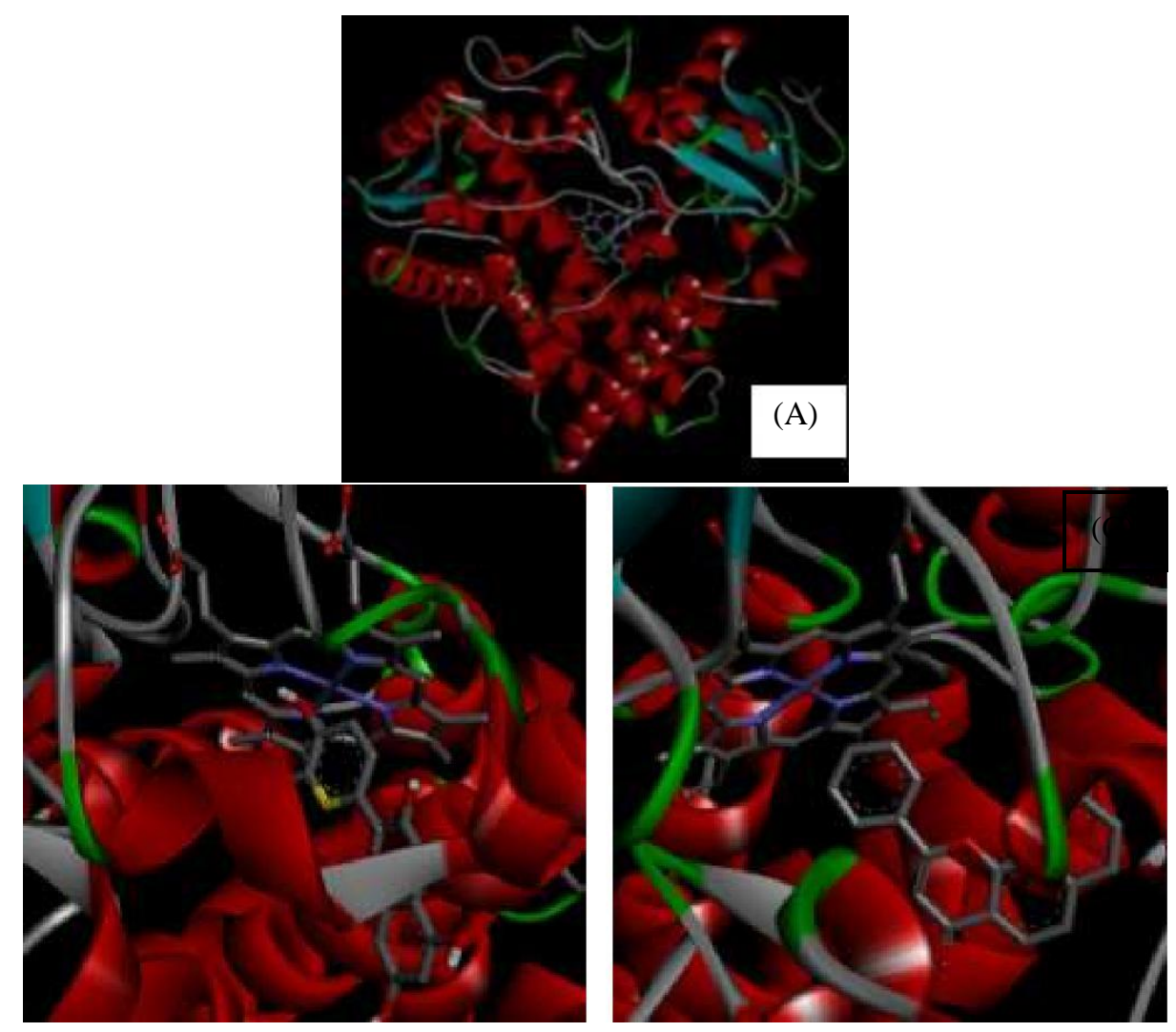

Figure 1. (A)3D structure of CYP1A1 (B)3D structure of CYP1A1-isorhamnetin (C)3D structure of CYP1A1- $\alpha$-naphthoflavone

Based on the analysis of 2D known that Isorhamnetin-CYP1A1 connected by the two strongest chemical bond that $\pi$ (second bonding). The existence the strongest hydrogen bond formed stable as indicated by the small free energy possessed both ligand-receptor complex.

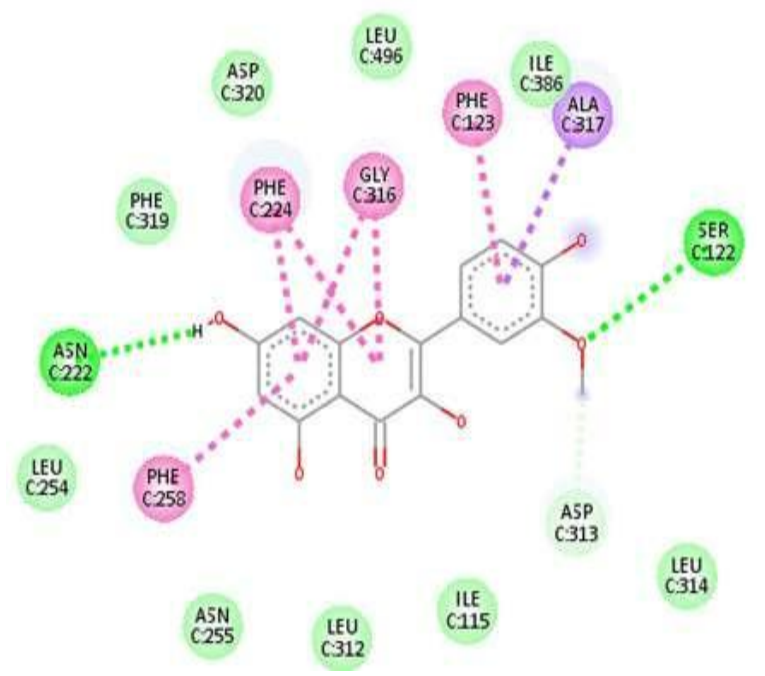

Figure 2. 2D picture of CYP1A1-isorhamnetin

CYP1A1 activity will affect the activity of c-JUN and c-FOS. JUN/FOS is a 
heterodimer protein that forms AP-1 structure. AP-1 plays roles in the formation of Matrix metalloproteinases effect on collagen production. Based on the pathway, it is known that isorhamnetin dependent on CYP1A1 affect the activity of c-JUN and c-Fos thus inhibiting AP- activation. c-FOS role in the activation of AP-1 in keratinocytes (most cells in the epidermis of the skin), so the inhibition of c-FOS would reduce possible damage to the cells in the epidermis. Besides inhibition of CYP1A1 will prevent c-Fos and c-JUN to interact so that lower expression of AP-1 cells on the skin epidermis and dermi. Decreased expression of AP-1 would inhibit the action of MMPs (Matrix metalloproteinases) so that damage to collagen and firoblast can be inhibited. It is able to reduce the risk of photoaging in human skin. 